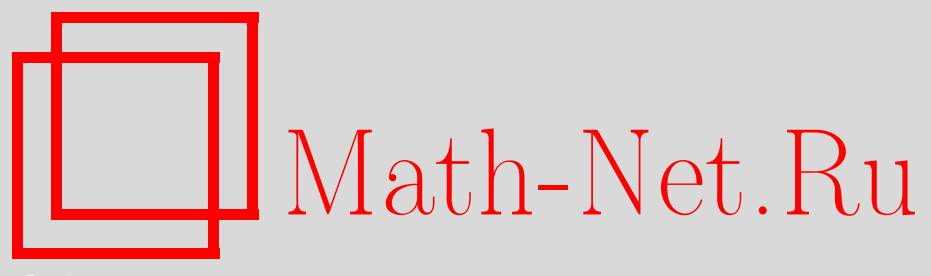

В. Г. Пузаренко, К. В. Сторожук, Компактность и сходимость по монотонным подсетям, Матем. заметки, 2015, том 97, выпуск 6, 945-946

DOI: https://doi.org/10.4213/mzm10498

Использование Общероссийского математического портала Math-Net.Ru подразумевает, что вы прочитали и согласны с пользовательским соглашением http://www . mathnet.ru/rus/agreement

Параметры загрузки:

IP: 54.166 .219 .16

26 апреля 2023 г., $17: 25: 04$

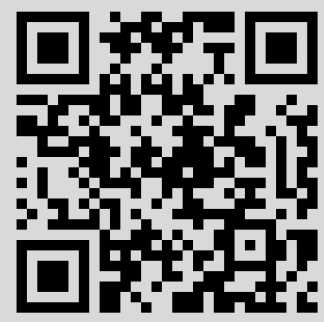




\section{Компактность и сходимость по монотонным подсетям}

\section{В. Г. Пузаренко, К. В. Сторожук}

1. Определения: направленности и сети. Понятие предела направленности, обобщающее обычное понятие предела последовательности, начиная с работ Мура и Смита [1] и Биркгофа [2], оказалось полезным в общей топологии, см., например, Келли [3] или Энгелькинг [4].

Направленное множество (направленностъ) - это частично упорядоченное множество $\left(\mathscr{A}, \leqslant_{A}\right)$ такое, что для любых $\alpha_{1}, \alpha_{2} \in \mathscr{A}$ найдется $\alpha_{3}$ такой, что $\alpha_{1} \leqslant{ }_{A} \alpha_{3}$ и $\alpha_{2} \leqslant{ }_{A} \alpha_{3}$. Поднаправленность $\left(\mathscr{A}, \leqslant_{A}\right)$ (или более тонкая направленность) - это направленность $\left(\mathscr{B}, \leqslant_{B}\right)$ вместе с отображением $\psi: \mathscr{B} \rightarrow \mathscr{A}$ таким, что значения $\psi(\beta)$, начиная с некоторого $\psi\left(\beta_{0}\right)$, становятся в $\mathscr{A}$ "сколь угодно велики", т.е.

$$
\forall \alpha_{0} \in \mathscr{A} \quad \exists \beta_{0} \in \mathscr{B} \quad \forall \beta \geqslant_{B} \beta_{0} \quad \psi(\beta) \geqslant_{A} \alpha_{0} .
$$

В частности, если $\mathscr{B} \subset \mathscr{A}$ - конфинальное подмножество, а $\psi$ - вложение, то говорят о монотонной поднаправленности.

Пусть $X$ - топологическое пространство. Сетъю в $X$ называют отображение вида $\mathscr{A} \rightarrow X$; записываем так: $\alpha \mapsto x_{\alpha}$. Пусть $\mathscr{B}$ - поднаправленность $\mathscr{A}$. (Часто вместо "сеть" говорят снова "направленность в $X$ ".) Подсетъю сети $x_{\alpha}$ называется отображение $\mathscr{B} \rightarrow X$ вида $\beta \mapsto x_{\psi(\beta)}$. Без риска непонимания пишем просто $x_{\beta}$.

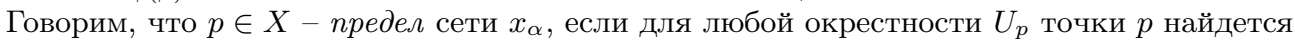
$\alpha_{0} \in \mathscr{A}$ такой, что $x_{\alpha} \in U_{p}$ для всех $\alpha \geqslant_{A} \alpha_{0}$. Точка $p \in X$ называется предельной точкой сети, если для каждой окрестности $U_{p}$ множество $\left\{\alpha \mid x_{\alpha} \in U_{p}\right\}$ конфинально в $\mathscr{A}$. Каждая предельная точка сети $\left\{x_{\alpha} \mid \alpha \in \mathscr{A}\right\}$ является пределом какой-то подсети $\left\{x_{\beta} \mid \beta \in \mathscr{B}\right\}$.

Известно, что $X$ компактно тогда и только тогда, когда любая сеть имеет сходящуюся подсеть. В этом утверждении нельзя добавить к слову “подсеть" слово "монотонная". Даже для последовательностей это не так. Например, $\{0,1\}^{c}$ компактно, но не секвенциально компактно [2; теорема 10].

Мы показываем, что сети без монотонных сходящихся подсетей бывают даже в числовом отрезке $X=I \subset \mathbb{R}$. Это легко следует из теоремы 1 . Правда, за простоту пространства $X$ приходится платить сложностью сети.

2. Сети без монотонных сходящихся подсетей. Пусть $\kappa$ - бесконечная мощность. Рассмотрим кардинал $2^{\kappa}$. Он вполне упорядочен как множество всех ординалов мощности меньшей $2^{\kappa}$.

Теорема 1. Пусть $X$ - топологическое пространство мощности $2^{\kappa}$ с точечным весом $\kappa$. Пусть отображение $\alpha \mapsto x_{\alpha}$ ингективно отображает $2^{\kappa}$ в X. Тогда никакая монотонная подсеть в $\left\{x_{\alpha}\right\}$ не имеет в $X$ предела.

ДокАзАтЕльство. Предположим, что $\{\beta\} \subset 2^{\kappa}$ - монотонная поднаправленность, сходящаяся к какому-то $p \in X$. Возьмем семейство окрестностей $\left\{U_{s} \mid s \in \kappa\right\}$ такое, что $\bigcap_{s \in k} U_{s}=\{p\}$. Для каждого $s \in \kappa$ найдется $\beta_{s}$ такой, что $x_{\beta} \in U_{s}$ для любого $\beta \geqslant \beta_{s}$.

Работа первого автора выполнена при финансовой поддержке программы "Ведущие научные школы” (грант № НШ-860.2014.1) и Российским фондом фундаментальных исследований (грант № 15-01-05144-a).

Работа второго автора выполнена при финансовой поддержке программы "Ведущие научные школы” (грант № НШ-2263.2014.1) и Российским фондом фундаментальных исследований (грант № 15-01-05929-a).

DOI: $10.4213 / \mathrm{mzm} 10498$ 
Подмножество $\left\{\beta_{s} \mid s \in \kappa\right\}$ не может быть конфинальным в $2^{\kappa}$ в силу $\kappa$-регулярности мощности $2^{\kappa}$. Поэтому существует $\beta<2^{\kappa}$ такое, что $\beta_{s} \leqslant \beta$ для всех $s \in \kappa$. Последнее означает, что $x_{\beta^{\prime}} \in \bigcap_{s \in k} U_{s}=\{p\}$ для каждого $\beta^{\prime}>\beta$ - противоречие с инъективностью. Теорема доказана.

Теорема 1 иллюстрирует топологическую “дикость" полных порядков. Например, если $\left(I, \tau_{1}\right)$ - порядковая топология, порожденная каким-то вполне упорядочением числового отрезка $I$, а $\tau_{2}$ - стандартная топология на $I$, то тождественное отображение $\left(I, \tau_{1}\right) \rightarrow$ $\left(I, \tau_{2}\right)$ разрывно на любом континуальном подмножестве.

Это можно также сравнить с тем известным фактом, что всякая непрерывная вещественная функция $f:\left[0, \omega_{1}\right) \rightarrow \mathbb{R}$ финально постоянна. Следующая теорема в некотором смысле двойственна теореме 1 .

Теорема 2. Если $X$ - топологическое пространство мощности $2^{\kappa}$, то любая сетъ в $X$, направленная порядком $2^{\kappa}$, имеет сходящуюся монотонную подсеть.

ДоказАтельство. Существует точка $x$, имеющая прообраз $\{\beta\} \subset 2^{\kappa}$ мощности $\kappa$. Ясно, что $\{\beta\}$ - конфинальная направленность в $2^{\kappa}$ и подсеть $x_{\beta}$ постоянна.

Для замкнутости изложения докажем здесь $\kappa$-регулярность $2^{\kappa}$. Пусть $A$-множество мощности $\kappa$. Поскольку $\kappa \times \kappa=\kappa$, множество $A$ можно считать объединением $\kappa$ дизъюнктных подмножеств $A_{\alpha} \subset A$, каждое мощности $\kappa$. Предположим, что $2^{A}$ можно покрыть семейством $\kappa$ штук неких $S_{\alpha}$, где каждое $S_{\alpha} \subset 2^{A}$ состоит строго меньше чем из $2^{\kappa}$ подмножеств $A$. Для каждого $\alpha$ среди подмножеств множества $A_{\alpha}$ найдется такое подмножество $B_{\alpha} \subset A_{\alpha}$, что $s_{\alpha} \cap A_{\alpha} \neq B_{\alpha}$ ни для каких $s_{\alpha} \in S_{\alpha}$. Определим подмножество $B \in 2^{A}$ так: $B=\bigcup_{\alpha} B_{\alpha}$. Множество $B \in 2^{A}$ не может лежать ни в каком семействе $S_{\alpha}$, ибо $B \cap A_{\alpha}=$ $B_{\alpha}$. Противоречие.

\section{СПИСОК ЦИТИРОВАННОЙ ЛИТЕРАТУРЫ}

[1] E. H. Moore, H. L. Smith, Amer. J. Math., 44:2 (1922), 102-121. [2] Birkhoff, Garrett, Ann. of Math. (2), 38:1 (1937), 39-56. [3] Дж. Л. Келли, Общая топология, Наука, М., 1981. [4] Р. Энгелькинг, Общая топология, Мир, М., 1986.

\section{В. Г. Пузаренко}

Институт математики

им. С. Л. Соболева СО РАН, г. Новосибирск;

Новосибирский государственный университет

E-mail: vagrig@math.nsc.ru

\section{К. В. Сторожук}

Институт математики

им. С. Л. Соболева СО РАН, г. Новосибирск;

Новосибирский государственный университет

E-mail: stork@math.nsc.ru
Поступило

02.06 .2014

Исправленный вариант

17.10.2014 